\title{
STEM CELLS
}

\section{Could it be TIME to abandon BMCs?}

Two phase I/II trials presented at the 2012

AHA Scientific Sessions and published in JAMA have shed new light on the effects of stem cell therapy in patients with myocardial infarction (MI). In the placebocontrolled TIME trial, the investigators assessed whether differential timing of bone marrow mononuclear cell (BMCs) delivery affected left ventricular function soon after MI (3-7 days). By contrast, in the POSEIDON trial, allogeneic and autologous bone marrow-derived mesenchymal stem cells (MSCs) at various doses were compared in patients with ischaemic cardiomyopathy several years after MI. The findings from these studies were mixed, but highlight additional areas for research.

The TIME trial was the third study on BMCs by the Cardiovascular Cell Therapy Research Network. The two earlier studies by this group produced negative results, and this latest trial has followed suit. In the era of rapid primary percutaneous coronary intervention, the study inclusion criterion of a postrevascularization left ventricular (LV) ejection fraction $\leq 45 \%$ was uncommon. Therefore, out of 3,347 screened patients, only 120 were included in the study. The cohort was predominantly male and of white ethnicity. Patients were randomly assigned to receive infusion of autologous BMCs $\left(150 \times 10^{6}\right.$ cells $)$ or a placebo into the infarct-related artery. Cell delivery was performed with $12 \mathrm{~h}$ of aspiration, and usually within $1 \mathrm{~h}$ of cell processing.

After 6 months, no significant differences between the BMC and placebo groups were evident for the primary (change in
LV ejection fraction and regional LV wall motion in the infarct or border zones) or secondary (major adverse cardiovascular events, and changes in LV volumes or infarct size) end points. The investigators postulate that these BMCs, harvested from patients with coronary heart disease, could have had reduced regenerative capacity as a result of impaired cytokine release or signalling.

The TIME trial was "well intentioned, nicely designed, and impeccably executed," according to Eduardo Marbán and Konstantinos Malliaris who wrote an accompanying Editorial. The results of the SWISS-AMI study, also presented at the AHA meeting but not yet published, support those of the TIME trial. Principle Investigator Jay Traverse concludes that "BMCs are a weak cell type in this population and offer little additional recovery."

In the POSEIDON trial, 30 patients ( $86.7 \%$ were male, $93.0 \%$ were white) with ischaemic cardiomyopathy secondary to MI were randomly allocated to undergo transendocardial injection of allogeneic $(n=15)$ or autologous $(n=15)$ MSCs at doses of either 20 million, 100 million, or 200 million cells $(n=5$ in each dose category). The primary safety end point of a serious adverse event within 30 days was experienced by one patient in each group (hospitalization for heart failure). No deaths occurred. The 1-year incidence of serious adverse events was $33.3 \%$ in the allogeneic group and $53.3 \%$ in the autologous group, although this difference did not reach statistical significance. Both cell types produced reverse LV remodelling, but this change did not translate into an

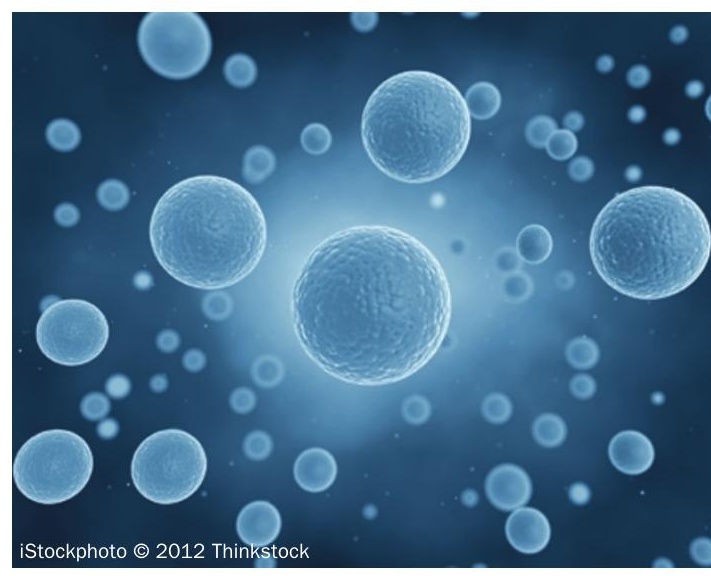

improvement in LV function. Autologous, but not allogeneic, MSCs improved patients' 6-min walk time, NYHA classification, and quality of life when compared with baseline. Interestingly, the lowest dose of cells (20 million) produced the greatest benefits. In the majority of patients, allogeneic cells did not cause a donor-specific immunological response. The investigators assert that their data "strongly support the ongoing development of allogeneic MSC therapy". In their Editorial, Marbán and Malliaris comment that "cautious optimism prevails for cells types other than [BMCs], including MSCs". However, therapeutic use of BMCs will be tested further in the upcoming European phase III BAMI trial.

Alexandra King 